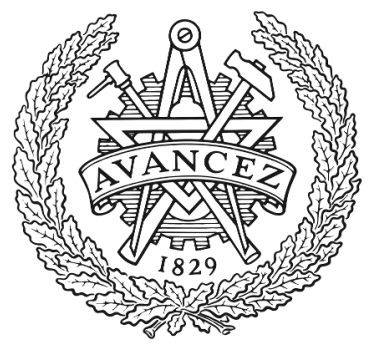

CHALMERS

UNIVERSITY OF TECHNOLOGY

\title{
High-Q Dispersion-Engineered Si3N4 Microresonators Based on a Subtractive Processing Technique
}

Downloaded from: https://research.chalmers.se, 2023-04-26 14:30 UTC

Citation for the original published paper (version of record):

Ye, Z., Twayana, K., Andrekson, P. et al (2020). High-Q Dispersion-Engineered Si3N4

Microresonators Based on a Subtractive Processing Technique. Optics InfoBase Conference Papers. http://dx.doi.org/10.1364/CLEO_AT.2020.JTh2F.24

N.B. When citing this work, cite the original published paper. 


\title{
High-Q Dispersion-Engineered $\mathrm{Si}_{3} \mathrm{~N}_{4}$ Microresonators Based on a Subtractive Processing Technique
}

\author{
Zhichao Ye*, Krishna Twayana, Peter A. Andrekson, and Victor Torres-Company ${ }^{* *}$ \\ Department of Microtechnology and Nanoscience, Chalmers University of Technology, 41296 Gothenburg, Sweden \\ "zhichao@chalmers.se, ${ }^{* *}$ torresv@chalmers.se
}

\begin{abstract}
We demonstrate dispersion-engineered $\mathrm{Si}_{3} \mathrm{~N}_{4}$ microresonators with mean $\mathrm{Q}$ values $>10$ million fabricated using standard subtractive techniques. Soliton microcombs at $100 \mathrm{GHz}$ line spacing and mode-locked dark-pulse Kerr combs are reported.
\end{abstract}

\section{Introduction}

Silicon nitride $\left(\mathrm{Si}_{3} \mathrm{~N}_{4}\right)$ has become a mature material for applications relying on the nonlinear Kerr effect because of its wide transparency window, absence of nonlinear loss in the near infrared and ability to engineer the waveguide dispersion. $\mathrm{Si}_{3} \mathrm{~N}_{4}$ microresonators constitute the cornerstone of the most advanced Kerr frequency combs (microcombs) reported to date. One critical parameter that dictates the performance of microcombs is the quality factor of the microresonator. Higher Qs (intrinsic Q > 10 million) are particularly relevant when considering co-integration of pump laser diodes with soliton microcombs operating at repetition rates compatible with high-speed electronics ( $100 \mathrm{GHz}$ and below) [1].

The fabrication process based on the Damascene reflow provides the highest-level of reproducibility in dispersion-engineered $\mathrm{Si}_{3} \mathrm{~N}_{4}$ microresonators [2]. In this fabrication technique, the $\mathrm{Si}_{3} \mathrm{~N}_{4}$ film is deposited on a silica preform that has been previously reflowed at high temperature. Intrinsic Qs in the order of 15 million have been recently reported, and microcombs with sub-100 GHz repetition rates demonstrated [3]. One drawback of this fabrication approach is that the aspect ratio dependent etching affects the uniformity of the waveguide height across the wafer, which is a potential issue if other photonic circuits besides microresonators are to be fabricated onto the same wafer. Traditionally, $\mathrm{Si}_{3} \mathrm{~N}_{4}$ photonic circuits are fabricated using a subtractive processing, where devices are patterned onto the as-deposited films (see e.g. [4,5]). These subtractive processing methods have no major problems with device uniformity, but the attainable Qs are limited by sidewall roughness, leading to a practical tradeoff between modal confinement (and therefore ability to engineer dispersion) and Q factor. Intrinsic Qs > 10 million have been reported before, but for waveguide geometries that are unoptimized for dispersion or for isolated resonances. Thus far, it has been unclear whether high Qs in dispersion-engineered $\mathrm{Si}_{3} \mathrm{~N}_{4}$ microresonators could be attained in a reproducible manner using standard processing techniques. Here, we show mean Qs > 10 million in $\mathrm{Si}_{3} \mathrm{~N}_{4}$ microresonators using a subtractive processing technique [6]. We also demonstrate for the first-time $\mathrm{Si}_{3} \mathrm{~N}_{4}$ soliton microcombs operating at $100 \mathrm{GHz}$ fabricated using standard processing techniques. The dispersion can be easily controlled, and we also demonstrate mode-locked dark-pulse Kerr combs. These results enhance the possibilities for co-integrating microcombs with advanced photonic integrated circuits based on ultra-low-loss $\mathrm{Si}_{3} \mathrm{~N}_{4}$ waveguides.

\section{Fabrication flow and statistical analysis of microresonators}

The fabrication process starts with an oxidized 3-inch Si wafer. The $3 \mu \mathrm{m}$ silica undercladding is patterned to stop the formation of cracks in the subsequent $\mathrm{Si}_{3} \mathrm{~N}_{4}$ layer [7]. A thick $\mathrm{Si}_{3} \mathrm{~N}_{4}$ film is deposited via low-pressure chemical vapor deposition in a two-step process. The target thickness is either $600 \mathrm{~nm}$ for normal-dispersion microcombs or $740 \mathrm{~nm}$ for anomalous. After annealing, electron beam lithography and dry etching by inductively coupled plasma reactive ion etching define the core waveguide structures. Specifics of the recipe are detailed in [6]. A TEOS $\mathrm{SiO}_{2}$ layer of 500 $\mathrm{nm}$ followed by $\sim 2 \mu \mathrm{m}$ of plasma-enhanced chemical vapor deposited silica forms the top cladding. Figure 1 (a) shows a scanning electron microscope (SEM) image of the core waveguide geometry. The line edge roughness is $1 \mathrm{~nm}$ in root mean square (close to the limit of what can be measured in our facilities) and $450 \mathrm{~nm}$ correlation length. The top surface of the $\mathrm{Si}_{3} \mathrm{~N}_{4}$ film is analyzed with an atomic force microscope, resulting in $0.18 \mathrm{~nm}$ rms roughness. Figure 1 (b) shows an SEM image of two waveguide structures placed in close proximity after focused ion beam milling, highlighting the absence of air voids thanks to the high conformal deposition of our $\mathrm{SiO}_{2}$ cladding structure.

Next, we fabricate microresonator structures. The transmission spectra of multiple structures are analyzed via scanning laser spectroscopy using a tunable external cavity laser diode. The tuning of the laser is calibrated with the aid of a self-referenced fs laser frequency comb [8]. In this way, the location of the longitudinal modes can be measured with great accuracy, allowing us to calculate their quality factor from a uniform spectral grid and in turn 
the microresonator dispersion. Figure 1(c) shows the histogram of multiple resonances measured across in the 1530$1610 \mathrm{~nm}$ range for microresonators featuring a cross-section geometry of $1850 \times 600 \mathrm{~nm}^{2}$ (width $\mathrm{x}$ height). The histogram is analyzed for all devices on the same chip having identical radius. The most probable value corresponds to an intrinsic linewidth of $15-18 \mathrm{MHz}$ (mean intrinsic Q 11.4 x 106). Few resonances with large intrinsic linewidth are observed due to avoided mode crossings.
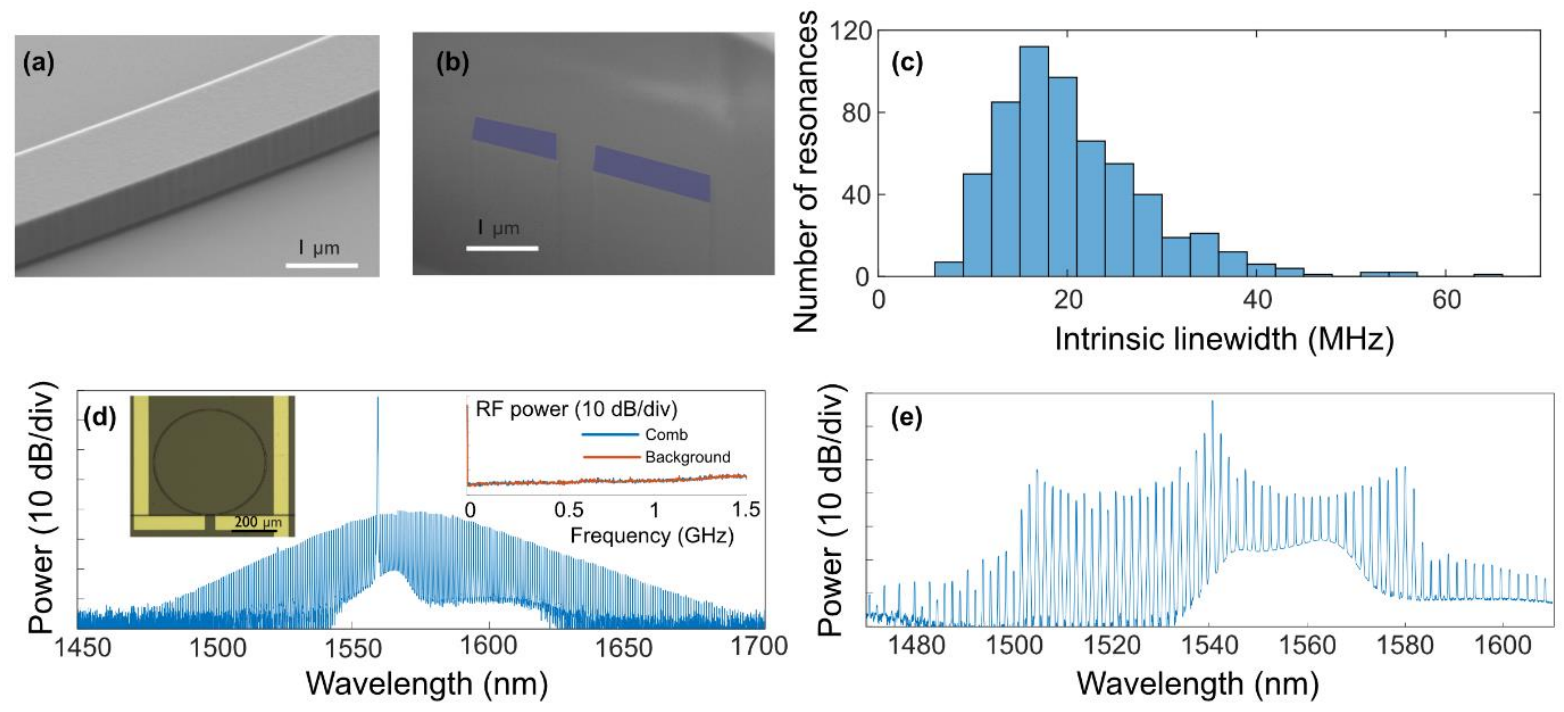

Fig. 1. (a) SEM image of $\mathrm{Si}_{3} \mathrm{~N}_{4}$ core waveguide. (b) $\mathrm{SEM}$ image of two $\mathrm{Si}_{3} \mathrm{~N}_{4}$ waveguides (purple) in close proximity. (c) Histogram of intrinsic linewidth of resonances for a $100 \mathrm{GHz}$ microresonators with cross-section geometry 1850 x $600 \mathrm{~nm}^{2}$. (d) Soliton microcomb at $100 \mathrm{GHz}$ repetition rate. Left inset displays a figure of the microresonator, where a thermo-optic heater is placed on top of the ring. Right inset indicates absence of amplitude noise in the soliton microcomb. (e) Mode-locked dark-pulse Kerr comb with $210 \mathrm{GHz}$ line spacing.

\section{Generation of coherent microcombs}

We test the possibility to generate microcombs with our $\mathrm{Si}_{3} \mathrm{~N}_{4}$ technology. In the first case, we engineer the crosssection of the waveguide to $2000 \times 740 \mathrm{~nm}^{2}$ and measure a group-velocity dispersion coefficient of $-67 \mathrm{ps}^{2} / \mathrm{km}$. The mean Q of this device is 12.5 million and the free spectral range (FSR) $100 \mathrm{GHz}$. We fabricate a thermo-optic heater on top of the ring to facilitate comb generation while pumping without tuning the pump wavelength [9]. When pumping with $\sim 100 \mathrm{~mW}$ of coupled power, we generate a low-noise microcomb as displayed in Fig. 1(d). This is the first time that a $100 \mathrm{GHz}$ soliton is generated in a $\mathrm{Si}_{3} \mathrm{~N}_{4}$ microresonator fabricated using a subtractive processing technique. The conversion efficiency is only $0.8 \%$ due to the naturally small duty cycle of the soliton pulse.

Our ability to engineer the dispersion allows us to investigate dark-pulse Kerr comb formation in normaldispersion high-Q microresonators [10]. We identify a ring resonator from the ones assessed in Fig. 1(c) that has a suitable mode-crossing around $1540 \mathrm{~nm}$, which facilitates phase-matching at the initial stage of comb formation [10]. When the device is pumped with $200 \mathrm{~mW}$ on-chip power, a coherent dark-pulse Kerr comb emerges. The measured conversion efficiency (output comb power excluding pump line divided by input power) is $23 \%$. The line spacing corresponds to twice the microresonator's FSR, leading us to conclude that the comb corresponds to two dark pulses circulating in the cavity.

We acknowledge support from the European Research Council (CoG 771410), the Swedish Research Council (2015-00535, 2016-03960, 201606077) and the H2020 Marie Curie Innovative Training Network Microcomb (812818).

\section{References}

[1] B. Stern et al., "Battery-operated integrated frequency comb generator," Nature 562, 401-405 (2018).

[2] J. Liu et al., "Ultralow-power chip-based soliton microcombs for photonic integration," Optica 5, 1347-1353 (2018).

[3] J. Liu et al., "Nanophotonic soliton-based microwave synthesizers", arXiv 1901.10372

[4] X. Ji et al., "Ultra-low-loss on-chip resonators with sub-milliwatt parametric oscillation threshold," Optica 4, 619-624 (2017).

[5] Y. Xuan et al., "High-Q silicon nitride microresonators exhibiting low-power frequency comb initiation," Optica 3, 1171-1180 (2016).

[6] Z. Ye et al., "High-Q Si3N4 microresonators based on a subtractive processing for Kerr nonlinear ...", Opt. Express 27, 35719-35727 (2019).

[7] K. Luke et al., "Overcoming Si3N4 film stress limitations for high quality factor ring resonators," Opt. Express 21, 22829-22833 (2013).

[8] P. Del'Haye et al., "Frequency comb assisted diode laser spectroscopy for measurement of ...," Nature Photon. 3, 529-533 (2009).

[9] C. Joshi et al., "Thermally controlled comb generation and soliton modelocking in microresonators," Opt. Lett. 41, 2565-2568 (2016).

[10] X. Xue et al., "Mode-locked dark pulse Kerr combs in normal-dispersion microresonators," Nature Photon. 9, 594-600 (2015). 